\title{
COARSE LIPSCHITZ EMBEDDINGS OF JAMES SPACES
}

\author{
F. NETILLARD
}

\begin{abstract}
We prove that, for $1<p \neq q<\infty$, there does not exist any coarse Lipschitz embedding between the two James spaces $J_{p}$ and $J_{q}$, and that, for $1<p<q<\infty$ and $1<r<\infty$ such that $r \notin\{p, q\}, J_{r}$ does not coarse Lipschitz embed into $J_{p} \oplus J_{q}$.
\end{abstract}

\section{INTRODUCTION}

Let $(M, d)$ and $(N, \delta)$ be two metric spaces and $f: M \rightarrow N$.

The map $f$ is said to be a coarse Lipschitz embedding if there exist $\theta, A, B>0$ such that

$$
\forall x, y \in M \quad d(x, y) \geq \theta \Rightarrow A d(x, y) \leq \delta(f(x), f(y)) \leq B d(x, y) .
$$

Then we say that $M$ coarse Lipschitz embeds into $N$.

R.C. James introduced in [5] a non-reflexive space defined by :

$$
J=\left\{x: \mathbb{N} \rightarrow \mathbb{R} \text { s.t. } x(n) \rightarrow 0 \text { and }\|x\|_{J}=\sup _{p_{1}<\ldots<p_{n}}\left(\sum_{i=1}^{n-1}\left|x\left(p_{i+1}\right)-x\left(p_{i}\right)\right|^{2}\right)^{\frac{1}{2}}<\infty\right\}
$$

We will use the following spaces (where $1<p<\infty$ ), which are variants of $J$ :

$J_{p}=\left\{x: \mathbb{N} \rightarrow \mathbb{R}\right.$ s.t. $x(n) \rightarrow 0$ and $\left.\|x\|_{J_{p}}=\sup _{p_{1}<\ldots<p_{n}}\left(\sum_{i=1}^{n-1}\left|x\left(p_{i+1}\right)-x\left(p_{i}\right)\right|^{p}\right)^{\frac{1}{p}}<\infty\right\}$

Like in the case of $J$, the codimension of $J_{p}$ in $J_{p}^{* *}$ is 1 .

In this respect, we precise that $J_{p}^{* *}$ can be seen as :

$$
J_{p}^{* *}=\left\{x: \mathbb{N} \rightarrow \mathbb{R} \text { s.t. } \sup _{p_{1}<\ldots<p_{n}}\left(\sum_{i=1}^{n-1}\left|x\left(p_{i+1}\right)-x\left(p_{i}\right)\right|^{p}\right)^{\frac{1}{p}}<\infty\right\}
$$

All those spaces were introduced in [11].

In 2007, N.J. Kalton and N.L. Randrianarivony [5] proved that, if $r \notin\left\{p_{1}, \ldots, p_{n}\right\}$ where $1 \leq p_{1}<p_{2}<\ldots<p_{n}<\infty$, then $\ell_{r}$ does not coarse Lipschitz embed into $\ell_{p_{1}} \oplus \ldots \oplus \ell_{p_{n}}$.

The aim of this article is to prove similar results for the $J_{p}$ spaces. One of the main obstacles is the lack of reflexivity, which was crucial in Kalton-Randrianarivony's

2010 Mathematics Subject Classification. Primary 46B20; Secondary 46B80 .

Key words and phrases. Banach spaces, James spaces, coarse Lipschitz embeddings. 
work. However, the James spaces have nice properties of asymptotic uniform smoothness and weak* asymptotic uniform convexity that we shall use (see [8] or [9] for the definitions). We shall not refer to these notions in our paper, but we will build concrete equivalent norms on $J_{p}$ that will serve our purpose. Some compactness arguments will also be used to deal with the extra dimension in $J_{p}^{* *}$.

This paper is organized as follows. In Section 2 we summarize the notation and terminology and we give the basic results. Section 3 contains the proof of the nonexistence of a coarse Lipschitz embedding between two James spaces $J_{p}$ and $J_{q}$ for $1<p \neq q<\infty$. At the end of this last section, we show that, for $1<p<q<\infty$ and $1<r<\infty$ such that $r \notin\{p, q\}, J_{r}$ does not coarse Lipschitz embed into $J_{p} \oplus J_{q}$.

\section{Preliminaries}

Notation 2.1. Let $e_{n}$ defined by $e_{n}(k)=\delta_{n, k}$ for $k \in \mathbb{N}$. The sequence $\left(e_{n}\right)_{n=0}^{\infty}$ is a Schauder basis of $J_{p}$ (where $p>1$ ).

Moreover, the sequence $\left(e_{n}^{*}\right)_{n=0}^{\infty}$ of the coordinate functionals associated with $\left(e_{n}\right)_{n=0}^{\infty}$ is a Schauder basis of $J_{p}^{*}$.

When $u$ and $v$ in $J_{p}$ have a consecutive and disjoint finite supports with respect to $\left(e_{n}\right)_{n=0}^{\infty}$, we will denote $u \prec v$.

Likewise, when $u^{*}$ and $v^{*}$ in $J_{p}^{*}$ have a consecutive and disjoint finite supports with respect to $\left(e_{n}^{*}\right)_{n=0}^{\infty}$, we will denote $u^{*} \prec v^{*}$.

We start with the construction of an ad'hoc equivalent norm on $J_{p}$. We follow the construction given in [10] for $J_{2}$.

Lemma 2.2. Let $x_{1}, \ldots, x_{n}$ in $J_{p}$ such that their supports are consecutive and finite with respect to the basis $\left(e_{n}\right)_{n=0}^{\infty}$. Then

$$
\left\|\sum_{i=1}^{n} x_{i}\right\|_{J_{p}}^{p} \leq\left(2^{p}+1\right) \sum_{i=1}^{n}\left\|x_{i}\right\|_{J_{p}}^{p} .
$$

Proof. For $x \in J_{p}$, we denote $\operatorname{supp}(x)=\left\{n \in \mathbb{N}, e_{n}^{*}(x) \neq 0\right\}$. Then we can find disjoint intervals in $\mathbb{N}, \llbracket p_{i}, p_{i}^{\prime} \rrbracket$, with $1 \leq i \leq n$ and $p_{i}^{\prime}<p_{i+1}$, such that:

$\forall 1 \leq i \leq n, \operatorname{supp}\left(x_{i}\right) \subset \llbracket p_{i}, p_{i}^{\prime} \rrbracket$ (for convenience, we fix $p_{1}=0$ et we denote $\left.p_{n+1}=\infty\right)$.

Let now $q_{1}<\ldots<q_{k}$ be an arbitrary sequence in $\mathbb{N}$. We must show that

$$
\sum_{j=1}^{k-1}\left|y\left(q_{j}\right)-y\left(q_{j+1}\right)\right|^{p} \leq\left(2^{p}+1\right) \sum_{i=1}^{n}\left\|x_{i}\right\|_{J_{p}}^{p},
$$

where $y=\sum_{i=1}^{n} x_{i}$.

There exist an increasing sequence $\left(j_{m}\right)_{m=1}^{l}$ in $\{1, \ldots, k\}$ with $j_{1}=1$, and an increasing sequence $\left(i_{m}\right)_{m=1}^{l}$ in $\{1, \ldots, n\}$ such that, for any $1 \leq m \leq l,\left\{q_{j_{m}}, \ldots, q_{j_{m+1}}-\right.$

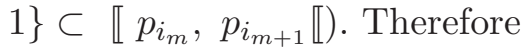

$$
\sum_{j=1}^{k-1}\left|y\left(q_{j}\right)-y\left(q_{j+1}\right)\right|^{p}=
$$




$$
\sum_{j=1}^{j_{2}-1}\left|y\left(q_{j}\right)-y\left(q_{j+1}\right)\right|^{p}+\left|y\left(q_{j_{2}-1}\right)-y\left(q_{j_{2}}\right)\right|^{p}+\ldots+\sum_{j=j_{l-1}}^{j_{l}-1}\left|y\left(q_{j}\right)-y\left(q_{j+1}\right)\right|^{p} .
$$

We have that

$$
\forall 1 \leq m \leq l-1, \sum_{j=j_{m}}^{j_{m+1}-1}\left|y\left(q_{j}\right)-y\left(q_{j+1}\right)\right|^{p} \leq\left\|x_{i_{m}}\right\|_{J_{p}}^{p} .
$$

And for all $2 \leq m \leq l-1$,

$\left|y\left(q_{j_{m}-1}\right)-y\left(q_{j_{m}}\right)\right|^{p} \leq 2^{p-1}\left|y\left(q_{j_{m}-1}\right)\right|^{p}+2^{p-1}\left|y\left(q_{j_{m}}\right)\right|^{p} \leq 2^{p-1}|| x_{i_{m}}\left\|_{J_{p}}^{p}+2^{p-1}|| x_{i_{m+1}}\right\|_{J_{p}}^{p}$.

Then

$\sum_{j=1}^{k-1}\left(y\left(q_{j}\right)-y\left(q_{j+1}\right)\right)^{p} \leq\left(2^{p-1}+1\right)\left\|x_{i_{1}}\right\|_{J_{p}}^{p}+\left(2^{p}+1\right)\left\|x_{i_{2}}\right\|_{J_{p}}^{p}+\ldots+\left(2^{p}+1\right)\left\|x_{i_{l-1}}\right\|_{J_{p}}^{p}+\left(2^{p-1}+1\right)\left\|x_{i_{l}}\right\|_{J_{p}}^{p}$.

This concludes the proof of our Lemma.

We now define a new norm on $J_{p}^{*}$ as follows. Let $q$ be the conjugate exponent of $p$. For $x^{*} \in J_{p}^{*}$, we set

$$
\left|x^{*}\right|_{J_{p}^{*}}=\sup \left\{\left(\sum_{i=1}^{n}\left\|x_{i}^{*}\right\|_{J_{p}^{*}}^{q}\right)^{\frac{1}{q}}: x^{*}=x_{1}^{*}+\ldots+x_{n}^{*} \text { et } x_{1}^{*} \prec \ldots \prec x_{n}^{*}\right\},
$$

where $\|.\|_{J_{p}^{*}}$ denotes the dual norm of $\|.\|_{J_{p}}$. We can now state the following proposition.

Proposition 2.3. The norm $|\cdot|_{J_{p}^{*}}$ is the dual norm of an equivalent norm on $J_{p}$ (that we shall denote $\left.|\cdot| J_{p}\right)$.

Moreover, $|.| J_{p}^{*}$ satisfies the following property: for any $x^{*}, y^{*}$ in $J_{p}^{*}$ such that $x^{*} \prec y^{*}$, we have that

$$
\left|x^{*}+y^{*}\right|_{J_{p}^{*}}^{q} \geq\left|x^{*}\right|_{J_{p}^{*}}^{q}+\left|y^{*}\right|_{J_{p}^{*}}^{q}
$$

Proof. To show that $|\cdot|_{J_{p}^{*}}$ is a norm, we only detail the proof of the triangle inequality : let $\left(x^{*}, y^{*}\right) \in\left(J_{p}^{*}\right)^{2}$ that we may assume with finite supports. Let now $u_{1}^{*} \prec u_{2}^{*} \prec \ldots \prec u_{n}^{*}$ in $J_{p}^{*}$ such that

$$
x^{*}+y^{*}=u_{1}^{*}+\ldots+u_{n}^{*}
$$

We write, for $i \in \llbracket 1, n \rrbracket, u_{i}^{*}=x_{i}^{*}+y_{i}^{*}$, where $x^{*}=\sum_{i=1}^{n} x_{i}^{*}$ and $y^{*}=\sum_{i=1}^{n} y_{i}^{*}$.

Thanks to the triangle inequality for $\|\cdot\|_{J_{p}^{*}}$, we get:

$$
\left(\sum_{i=1}^{n}\left\|u_{i}^{*}\right\|_{J_{p}^{*}}^{q}\right)^{\frac{1}{q}} \leq\left(\sum_{i=1}^{n}\left(\left\|x_{i}^{*}\right\|_{J_{p}^{*}}+\left\|y_{i}^{*}\right\|_{J_{p}^{*}}\right)^{q}\right)^{\frac{1}{q}} .
$$

It then follows from Minkowski's inequality that 


$$
\left(\sum_{i=1}^{n}\left\|u_{i}^{*}\right\|_{J_{p}^{*}}^{q}\right)^{\frac{1}{q}} \leq\left(\sum_{i=1}^{n}\left\|x_{i}^{*}\right\|_{J_{p}^{*}}^{q}\right)^{\frac{1}{q}}+\left(\sum_{i=1}^{n}\left\|y_{i}^{*}\right\|_{J_{p}^{*}}^{q}\right)^{\frac{1}{q}} \leq\left|x^{*}\right|_{J_{p}^{*}}+\left|y^{*}\right|_{J_{p}^{*}} .
$$

We have shown that the triangle inequality is valid for $|\cdot|_{p}^{*}$.

Next we show that there exists $c>0$ (which will be detailed later) such that, for $x_{1}^{*}, \ldots, x_{n}^{*}$ in $J^{*}$ satisfying $x_{1}^{*} \prec \ldots \prec x_{n}^{*}$ with respect to the basis $\left(e_{n}^{*}\right)_{n=0}^{\infty}$ :

$$
\left\|\sum_{i=1}^{n} x_{i}^{*}\right\|_{J_{p}^{*}}^{q} \geq c \sum_{i=1}^{n}\left\|x_{i}^{*}\right\|_{J_{p}^{*}}^{q}
$$

So, let $x_{1}^{*} \prec \ldots \prec x_{n}^{*}$, with, for $i \in \llbracket 1, n \rrbracket, \operatorname{supp}\left(x_{i}^{*}\right) \subseteq \llbracket p_{i}, q_{i} \rrbracket$, where $q_{i}<p_{i+1}$ for $i \in \llbracket 1, n-1 \rrbracket$. Fix now $\varepsilon>0$.

Since $\left(e_{i}\right)_{i=0}^{\infty}$ is a monotone basis:

$$
\exists x_{i} \in J_{p},\left\{\begin{aligned}
x_{i}^{*}\left(x_{i}\right) & \geq\left\|x_{i}^{*}\right\|_{J_{p}^{*}}^{q}-\varepsilon \\
\left\|x_{i}\right\|_{J_{p}} & \leq 2\left\|x_{i}^{*}\right\|_{J_{p}^{*}}^{q-1} \\
\operatorname{supp}\left(x_{i}\right) & \subseteq \llbracket p_{i}, q_{i} \rrbracket
\end{aligned}\right.
$$

Thanks to Lemma 2.2 : $\exists C>0,\left\|\sum_{i=1}^{n} x_{i}\right\|_{J_{p}}^{p} \leq C \sum_{i=1}^{n}\left\|x_{i}\right\|_{J_{p}}^{p}\left(\right.$ where $\left.C=2^{p}+1\right)$.

Since $\|.\|_{J_{p}^{*}}$ is the dual norm of $\|\cdot\|_{J_{p}}$, we have that

$$
\left\|\sum_{i=1}^{n} x_{i}^{*}\right\|_{J_{p}^{*}} \geq\left(\sum_{i=1}^{n} x_{i}^{*}\right)\left(\sum_{i=1}^{n} x_{i}\right)\left(\left\|\sum_{i=1}^{n} x_{i}\right\|_{J_{p}}\right)^{-1}
$$

and

$\left\|\sum_{i=1}^{n} x_{i}^{*}\right\|_{J_{p}^{*}} \geq\left(\sum_{i=1}^{n} x_{i}^{*}\left(x_{i}\right)\right)\left(\left\|\sum_{i=1}^{n} x_{i}\right\|_{J_{p}}\right)^{-1} \geq\left(\sum_{i=1}^{n}\left\|x_{i}^{*}\right\|_{J_{p}^{*}}^{q}-\varepsilon\right)\left(C^{\frac{1}{p}}\left(\sum_{i=1}^{n}\left\|x_{i}\right\|_{J_{p}}^{p}\right)^{\frac{1}{p}}\right)^{-1}$.

Moreover, $\left(\sum_{i=1}^{n}\left\|x_{i}\right\|_{J_{p}}^{p}\right)^{\frac{1}{p}} \leq 2\left(\sum_{i=1}^{n}\left\|x_{i}^{*}\right\|_{J_{p}^{*}}^{p(q-1)}\right)^{\frac{1}{p}}=2\left(\sum_{i=1}^{n}\left\|x_{i}^{*}\right\|_{J_{p}^{*}}^{q}\right)^{\frac{1}{p}}$.

Letting $\varepsilon$ tend to 0 , we obtain :

$$
\left\|\sum_{i=1}^{n} x_{i}^{*}\right\|_{J_{p}^{*}} \geq \frac{1}{2 C^{\frac{1}{p}}}\left(\sum_{i=1}^{n}\left\|x_{i}^{*}\right\|_{J_{p}^{*}}^{q}\right)^{1-\frac{1}{p}}=\frac{1}{2 C^{\frac{1}{p}}}\left(\sum_{i=1}^{n}\left\|x_{i}^{*}\right\|_{J_{p}^{*}}^{q}\right)^{\frac{1}{q}} .
$$

So, we have established inequality (2.1) with $c=\frac{1}{2^{q} C^{\frac{q}{p}}}=\frac{1}{2^{q} C^{q-1}}$.

It follows easily that

$$
\left\|\left.x^{*}\right|_{J_{p}^{*}} \leq\left|x^{*}\right|_{J_{p}^{*}} \leq c^{-1 / q}\right\| x^{*} \|_{J_{p}^{*}} .
$$

Moreover, $|\cdot|_{J_{p}^{*}}$ is the dual norm of an equivalent norm on $J_{p}$. Indeed, it is clear that |. $\left.\right|_{J_{p}^{*}}$ is $\sigma\left(J_{p}^{*}, J_{p}\right)$ lower semi-continuous. 
Finally, it follows clearly from the definition of $|\cdot|_{J_{p}^{*}}$ that for all $x^{*}, y^{*}$ in $J_{p}^{*}$ such that $x^{*} \prec y^{*}$, we have that

$$
\left|x^{*}+y^{*}\right|_{J_{p}^{*}}^{q} \geq\left|x^{*}\right|_{J_{p}^{*}}^{q}+\left|y^{*}\right|_{J_{p}^{*}}^{q}
$$

Corollary 2.4. The dual norm $|\cdot|_{J_{p}^{* *}}$ of $|\cdot|_{J_{p}^{*}}$ satisfies the following property.

For $x \in J_{p}$ with a finite support and $y \in J_{p}^{* *}$ (not necessarily with finite support) such that $x \prec y$, we have

$$
|x+y|_{J_{p}^{* *}}^{p} \leq|x|_{J_{p}^{* *}}^{p}+|y|_{J_{p}^{* *}}^{p} .
$$

Proof. Let $x \in J_{p}$ which has a finite support and $y \in J_{p}^{* *}$ such that $x \prec y$, with $\left.\operatorname{supp}(x) \subset \llbracket m, n \rrbracket, \operatorname{supp}(y) \subset \llbracket m^{\prime},+\infty\right)$ and $n<m^{\prime}$. Fix $\varepsilon>0$.

There exists $z^{*} \in J_{p}^{*}$ such that

$$
\left|z^{*}\right|_{J_{p}^{*}}=|x+y|_{J_{p}^{* *}}^{p-1} \text { and } z^{*}(x+y) \geq|x+y|_{J_{p}^{* *}}^{p}-\varepsilon .
$$

Moreover, we can write $z^{*}=x^{*}+y^{*}$, with $x^{*} \prec y^{*}, z^{*}(x)=x^{*}(x)$ and $z^{*}(y)=y^{*}(y)$. We deduce that $|x+y|_{J_{p}^{* *}}^{p} \leq x^{*}(x)+y^{*}(y)+\varepsilon$. Then Hölder's inequality and Proposition 2.3 yield

$|x+y|_{J_{p}^{* *}}^{p} \leq\left(\left|x^{*}\right|_{J_{p}^{*}}^{q}+\left|y^{*}\right|_{J_{p}^{*}}^{q}\right)^{\frac{1}{q}}\left(|x|_{J_{p}^{* *}}^{p}+|y|_{J_{p}^{* *}}^{p}\right)^{\frac{1}{p}}+\varepsilon \leq\left(\left|x^{*}+y^{*}\right|_{J_{p}^{*}}\right)\left(|x|_{J_{p}^{* *}}^{p}+|y|_{J_{p}^{* *}}^{p}\right)^{\frac{1}{p}}+\varepsilon$.

Since $\left|z^{*}\right|_{J_{p}^{*}}=|x+y|_{J_{p}^{* *}}^{p-1}$, we get

$$
|x+y|_{J_{P}^{* *}}^{p} \leq\left(|x+y|_{J_{p}^{* *}}^{p-1}\right)\left(|x|_{J_{P}^{* *}}^{p}+|y|_{J_{p}^{* *}}^{p}\right)^{\frac{1}{p}}+\varepsilon .
$$

This finishes our proof.

We now turn to the study of the coarse Lipschitz embeddings between James spaces. Let us first recall some notation.

Definition 2.5. Let $(M, d)$ and $(N, \delta)$ be two metric spaces and $f: M \rightarrow N$ be a mapping. If $(M, d)$ is unbounded, we define

$\forall s>0, \operatorname{Lip}_{s}(f)=\sup \left\{\frac{\delta((f(x), f(y))}{d(x, y)}, d(x, y) \geq s\right\}$ and $\operatorname{Lip}_{\infty}(f)=\inf _{s>0} \operatorname{Lip}_{s}(f)$.

Note that $f$ is coarse Lipschitz if and only if $\operatorname{Lip}_{\infty}(f)<\infty$.

We also recall a classical definition.

Definition 2.6. Given a metric space $X$, two points $x, y \in X$, and $\delta>0$, the approximate metric midpoint set between $x$ and $y$ with error $\delta$ is the set :

$$
\operatorname{Mid}(x, y, \delta)=\left\{z \in X: \max \{d(x, z), d(y, z)\} \leq(1+\delta) \frac{d(x, y)}{2}\right\}
$$

The use of approximate metric midpoints in the study of nonlinear geometry is due to Enflo in an unpublished paper and has been used elsewhere, e.g. 2], 3] and [6]. The next proposition and its proof can be found for instance in [8] and [9]. 
Proposition 2.7. Let $X$ be a normed space and suppose $M$ is a metric space. Let $f: X \rightarrow M$ be a coarse Lipschitz map. If Lip $p_{\infty}(f)>0$, then for any $t, \varepsilon>0$ and any $0<\delta<1$, there exist $x, y \in X$ with $\|x-y\|>t$ and

$$
f(\operatorname{Mid}(x, y, \delta)) \subset \operatorname{Mid}(f(x), f(y),(1+\varepsilon) \delta) .
$$

Let us now recall the definition of the metric graphs introduced in [8] that will be crucial in our proofs.

Notation 2.8. Let $\mathbb{M}$ be an infinite subset of $\mathbb{N}$ and $k \in \mathbb{N}$. We denote

$$
G_{k}(\mathbb{M})=\left\{\bar{n}=\left(n_{1}, \ldots, n_{k}\right), n_{i} \in \mathbb{M} \quad n_{1}<\ldots<n_{k}\right\} .
$$

Then we equip $G_{k}(\mathbb{M})$ with the distance $d(\bar{n}, \bar{m})=\left|\left\{j, n_{j} \neq m_{j}\right\}\right|$.

We end these preliminaries by recalling Ramsey's theorem and one of its immediate corollaries (see [4] for instance).

Theorem 2.9. Let $k, r \in \mathbb{N}$ and $f: G_{k}(\mathbb{N}) \rightarrow\{1, \ldots, r\}$ be any map. Then there exists an infinite subset $\mathbb{M}$ of $\mathbb{N}$ and $i \in\{1, \ldots, r\}$ such that, for every $\bar{n} \in G_{k}(\mathbb{M})$, $f(\bar{n})=i$.

Corollary 2.10. Let $(K, d)$ be a compact metric space, $k \in \mathbb{N}$ and $f: G_{k}(\mathbb{N}) \rightarrow K$. Then for every $\epsilon>0$, there exist an infinite subset $\mathbb{M}$ of $\mathbb{N}$ such that for every $\bar{n}, \bar{m} \in G_{k}(\mathbb{M}), d(f(\bar{n}), f(\bar{m}))<\epsilon$.

\section{The MAin Results}

Our first Lemma gives a description of approximate metric midpoints in $J_{p}$ that is analogous to situation in $\ell_{p}$ (see [8] or [9]). However, we need to use both the original and our new norm on $J_{p}$.

Lemma 3.1. Let $1<p<\infty$. We denote $E_{N}$ the closed linear span of $\left\{e_{i}, i>N\right\}$. Let now $x, y \in J_{p}, \delta \in(0,1), u=\frac{x+y}{2}$ and $v=\frac{x-y}{2}$. Then

(i) There exists $N \in \mathbb{N}$ such that:

$$
u+\delta^{\frac{1}{p}}|v|_{J_{p}} B_{\left(E_{N},|\cdot|_{J_{p}}\right)} \subset M i d_{|\cdot|_{J_{p}}}(x, y, \delta) .
$$

(ii) There is a compact subset $K$ of $J_{p}$ such that:

$$
\operatorname{Mid}_{\|\cdot\|_{J_{p}}}(x, y, \delta) \subset K+2 \delta^{\frac{1}{p}}\|v\|_{J_{p}} B_{\left(J_{p},\|\cdot\|_{J_{p}}\right)} .
$$

Proof. Fix $\lambda>0$.

Let $N \in \mathbb{N}$ such that $\left|v-v_{N}\right|_{J_{p}} \leq \lambda|v|_{J_{p}}$ and $\left|v_{N}\right|_{J_{p}}^{p} \geq\left(1+\lambda^{p}\right)^{-1}|v|_{J_{p}}^{p}$, where $v_{N}=\sum_{i=1}^{N} v(i) e_{i}$.

(i) Let now $z \in E_{N}$ so that $|z|_{J_{p}}^{p} \leq \delta|v|_{J_{p}}^{p}$. Then

$$
|x-(u+z)|_{J_{p}}^{p}=|v-z|_{J_{p}}^{p}=\left|v-v_{N}+v_{N}-z\right|_{J_{p}}^{p}
$$

It follows from the Corollary 2.4 that:

$$
|x-(u+z)|_{J_{p}}^{p} \leq\left|v-v_{N}-z\right|_{J_{p}}^{p}+\left|v_{N}\right|_{J_{p}}^{p} \leq\left(\left|v-v_{N}\right|_{J_{p}}+|z|_{J_{p}}\right)^{p}+|v|_{J_{p}}^{p}
$$


Therefore

$$
|x-(u+z)|_{J_{p}}^{p} \leq\left(\left(1+\delta^{1 / p}\right)^{p}+1\right)|v|_{J_{p}}^{p} \leq(1+\delta)^{p}|v|_{J_{p}}^{p},
$$

if $\lambda$ was chosen initially small enough.

We argue similarly to show that $|y-(u+z)|_{J_{p}}=|v+z|_{J_{p}} \leq(1+\delta)|v|_{J_{p}}$ and deduce that $u+z \in \operatorname{Mid}(x, y, \delta)$.

(ii) Fix $\nu>0$ and choose $N \in \mathbb{N}$ such that $\left\|v_{N}\right\|_{J_{p}}^{p} \geq\left(1-\nu^{p}\right)\|v\|^{p}$. We assume now that $u+z \in M i d_{\|\cdot\|_{J_{p}}}(x, y, \delta)$ and write $z=z^{\prime}+z^{\prime \prime}$ with $z^{\prime} \in F_{N}=\operatorname{sp}\left\{e_{i}, i \leq N\right\}$ and $z^{\prime \prime} \in E_{N}$.

Since $\|v-z\|\left\|_{J_{p}},\right\| v+z\left\|_{J_{p}} \leq(1+\delta)\right\| v \|_{J_{p}}$, we get, by convexity, that

$$
\left\|z^{\prime}\right\|_{J_{p}} \leq\|z\|_{J_{p}} \leq(1+\delta)\|v\|_{J_{p}} .
$$

Therefore, $u+z^{\prime}$ belongs to the compact set $K=u+(1+\delta)\|v\|_{J_{p}} B_{\left(F_{N},\|.\| J_{p}\right)}$. Moreover, for any $(m, n) \in\left(\mathbb{N}^{*}\right)^{2}$, with $m>n$ :

$$
\begin{aligned}
\max \left\{|v(n)-v(m)|^{p},|z(n)-z(m)|^{p}\right\} \leq & \frac{1}{2}\left(|(v(n)-z(n))-(v(m)-z(m))|^{p}\right. \\
& \left.+|(v(n)+z(n))-(v(m)+z(m))|^{p}\right) .
\end{aligned}
$$

Therefore

$$
\left(1-\nu^{p}\right)\|v\|_{J_{p}}^{p}+\left\|z^{\prime \prime}\right\|_{J_{p}}^{p} \leq\left\|v_{N}\right\|_{J_{p}}^{p}+\left\|z^{\prime \prime}\right\|_{J_{p}}^{p} \leq \frac{1}{2}\left(\|v-z\|_{J_{p}}^{p}+\|v+z\|_{J_{p}}^{p}\right) \leq(1+\delta)^{p}\|v\|_{J_{p}}^{p} \text {. }
$$

Then, if $\nu$ was chosen small enough, we get

$$
\left\|z^{\prime \prime}\right\|_{J_{p}}^{p} \leq\left[(1+\delta)^{p}-\left(1-\nu^{p}\right)\right]\|v\|_{J_{p}}^{p} \leq 2^{p} \delta\|v\|_{J_{p}}^{p} .
$$

Proposition 3.2. Let $1<p<q<\infty$ and $f:\left(J_{q},\left.|\cdot|\right|_{J_{q}}\right) \rightarrow\left(J_{p},\|\cdot\|_{J_{p}}\right)$ be a coarse Lipschitz embedding. Then, for any $t>0$ and for any $\varepsilon>0$, there exist $u \in J_{q}$, $\theta>t, N \in \mathbb{N}$ and $K$ a compact subset of $J_{p}$ such that

$$
f\left(u+\theta B_{\left(E_{N},|\cdot| J_{q}\right)}\right) \subset K+\varepsilon \theta B_{\left(J_{p},\|\cdot\|_{J_{p}}\right)} .
$$

Proof. If $\operatorname{Lip}_{\infty}(f)=0$, the conclusion is clear. So we assume that $\operatorname{Lip}_{\infty}(f)>0$. We choose a small $\delta>0$ (to be detailed later). Then we choose $s$ large enough so that $\operatorname{Lip}_{s}(f) \leq 2 \operatorname{Lip} \infty(f)$.

Then, by Proposition 2.7,

$$
\exists x, y \in J_{q},|x-y|_{J_{q}} \geq s \text { and } f\left(M i d_{|\cdot| J_{q}}(x, y, \delta)\right) \subset M i d_{|| \cdot \|_{J_{p}}}(f(x), f(y), 2 \delta) .
$$

Denote $u=\frac{x+y}{2}, v=\frac{x-y}{2}$ and $\theta=\delta^{\frac{1}{q}}|v|_{J_{q}}$. By Lemma 3.1, there exists $N \in \mathbb{N}$ such that $u+\theta B_{\left(E_{N},|\cdot|_{J_{q}}\right)} \subset M i d_{|\cdot|_{J_{q}}}(x, y, \delta)$ and there exists a compact subset $K$ of $J_{p}$ so that $\operatorname{Mid}_{\|\cdot\| \|_{J_{p}}}(f(x), f(y), 2 \delta) \subset K+2(2 \delta)^{\frac{1}{p}}\|f(x)-f(y)\|_{J_{p}} B_{\left(J_{p},\|\cdot\|_{J_{p}}\right)}$. But :

$$
\begin{aligned}
2(2 \delta)^{\frac{1}{p}}\|f(x)-f(y)\|_{J_{p}} & \leq 4 \operatorname{Lip}_{\infty}(f)(2 \delta)^{\frac{1}{p}}|x-y|_{J_{q}} \\
& \leq 8 \operatorname{Lip}_{\infty}(f) 2^{\frac{1}{p}} \delta^{\frac{1}{p}-\frac{1}{q}} \theta \leq \varepsilon \theta,
\end{aligned}
$$

if $\delta$ was chosen initially small enough. 
Then an appropriate choice of a large $s$ will ensure that $\theta \geq \frac{1}{2} \delta^{\frac{1}{q}} s>t$. This finishes the proof.

Corollary 3.3. Let $1<p<q<\infty$.

Then $J_{q}$ does not coarse Lipschitz embed into $J_{p}$.

Proof. We proceed by contradiction and suppose that there exists a coarse Lipschitz embedding $f:\left(J_{q},|\cdot|_{J_{q}}\right) \rightarrow\left(J_{p},\|\cdot\|_{J_{p}}\right)$.

With the notation of the previous proposition, we can find a sequence $\left(u_{n}\right)_{n=1}^{\infty}$ in $u+\theta B_{\left(E_{N},|\cdot|_{J_{q}}\right)}$, such that $\left|u_{n}-u_{m}\right|_{J_{q}} \geq \theta$ for $n \neq m$. Then $f\left(u_{n}\right)=k_{n}+\varepsilon \theta v_{n}$, with $k_{n} \in K$ et $v_{n} \in B_{\left(J_{p},\|.\|_{J_{p}}\right)}$. Since $K$ is compact, by extracting a subsequence, we may assume that $\left\|f\left(u_{n}\right)-f\left(u_{m}\right)\right\|_{J_{p}} \leq 3 \varepsilon \theta$.

Since $\varepsilon$ can be chosen arbitrarily small and $\theta$ arbitrarily large, this yields a contradiction.

In order to treat the coarse Lipschitz embeddability in the other direction, we shall use the Kalton-Randrianarivony graphs and some special subsets of them that we introduce now.

Definition 3.4. Let $\bar{n}, \bar{m} \in G_{k}(\mathbb{M})$ (where $\mathbb{M}$ is an infinite subset of $\mathbb{N}$ ).

We say that $(\bar{n}, \bar{m}) \in I_{k}(\mathbb{M})$ if $n_{1}<m_{1}<n_{2}<m_{2}<\ldots<n_{k}<m_{k}$.

Proposition 3.5. Let $\varepsilon>0$ and $f: G_{k}(\mathbb{N}) \rightarrow\left(J_{p}^{* *},||_{J_{p}^{* *}}\right)$ be a Lipschitz map.

Then, for any infinite subset $\mathbb{M}$ of $\mathbb{N}$, there exists $(\bar{n}, \bar{m}) \in I_{k}(\mathbb{M})$ such that

$$
|f(\bar{n})-f(\bar{m})|_{J_{p}^{* *}} \leq 2 \operatorname{Lip}(f) k^{\frac{1}{p}}+\varepsilon
$$

Proof. We shall prove this statement by induction on $k \in \mathbb{N}$

The proposition is clearly true for $k=1$.

Assume now that it is true for $k-1 \geq 1$.

Let $f: G_{k}(\mathbb{M}) \rightarrow J_{p}^{* *}$ be a Lipschitz map and $\varepsilon>0$.

Let $\eta>0$ (small enough : to be detailed later).

By a diagonal extraction process and thanks to the weak*-compactness, we can find an infinite subset $\mathbb{M}_{1}$ of $\mathbb{N}$ such that

$$
\forall \bar{n} \in G_{k-1}(\mathbb{N}), w^{*}-\lim _{k \in \mathbb{M}_{1}} f(\bar{n}, k)=g(\bar{n}) \in J_{p}^{* *}
$$

Then $\operatorname{Lip}(g) \leq \operatorname{Lip}(f)$, by weak*-lower semicontinuity of $|\cdot|_{J_{p}^{* *}}$.

Denote $g(\bar{n})=v(\bar{n})+c_{\bar{n}} \mathbb{I}$ (where $v(\bar{n}) \in J_{p}, c_{\bar{n}} \in \mathbb{R}$ and $\mathbb{I}$ is the constant sequence $(1,1,1, \ldots))$.

By Ramsey's theorem, there exists an infinite subset $\mathbb{M}_{2}$ of $\mathbb{M}_{1}$ such that

$$
\forall \bar{n}, \bar{m} \in G_{k-1}\left(\mathbb{M}_{2}\right) \quad\left|c_{\bar{n}}-c_{\bar{m}}\right|=|(v(\bar{n})-v(\bar{m}))-(g(\bar{n})-g(\bar{m}))|_{J_{p}^{* *}}<\eta .
$$

For $\bar{n}, \bar{m} \in G_{k-1}\left(\mathbb{M}_{2}\right)$ and $t, l \in \mathbb{M}_{2}$, set

$$
u_{\bar{n}, \bar{m}, t, l}=f(\bar{n}, t)-g(\bar{n})+g(\bar{m})-f(\bar{m}, l) .
$$

Using (3.2) and Corollary 2.4 we deduce that there exists $l_{0} \in \mathbb{N}$ such that for all $t, l \in \mathbb{M}_{2} \cap\left[l_{0},+\infty[:\right.$ 


$$
\left|v(\bar{n})-v(\bar{m})+u_{\bar{n}, \bar{m}, t, l}\right|^{p} \leq|v(\bar{n})-v(\bar{m})|^{p}+\left|u_{\bar{n}, \bar{m}, t, l}\right|^{p}+\eta .
$$

Then it follows from (3.3) that for all $t, l \in \mathbb{M}_{2} \cap\left[l_{0},+\infty[\right.$ :

$$
|f(\bar{n}, t)-f(\bar{m}, l)|_{J_{p}^{* *}}^{p} \leq\left|u_{\bar{n}, \bar{m}, t, l}\right|_{J_{p}^{* *}}^{p}+\left(|v(\bar{n})-v(\bar{m})|_{J_{p}}+\eta\right)^{p}+\eta .
$$

Moreover : $f(\bar{n}, t)-g(\bar{n})=w^{*}-\lim _{i}(f(\bar{n}, t)-f(\bar{n}, i))$.

Therefore, by weak*-lower semicontinuity of $|\cdot|_{J_{p}^{* *}}:|f(\bar{n}, t)-g(\bar{n})|_{J_{p}^{* *}} \leq \operatorname{Lip}(f)$.

Likewise : $|f(\bar{m}, l)-g(\bar{m})|_{J_{p}^{* *}} \leq \operatorname{Lip}(f)$.

Then, we deduce the following inequality : $\left|u_{\bar{n}, \bar{m}, t, l}\right|_{J_{p}^{* *}}^{p} \leq 2^{p} \operatorname{Lip}(f)^{p}$.

On the other hand, it follows from our induction hypothesis that:

$$
\exists(\bar{n}, \bar{m}) \in\left(I_{k-1}\left(\mathbb{M}_{2}\right)\right)^{2},|g(\bar{n})-g(\bar{m})|_{J_{p}^{* *}} \leq 2 \operatorname{Lip}(f)(k-1)^{\frac{1}{p}}+\eta .
$$

Then, for $t, l \in \mathbb{M}_{2} \cap\left[l_{0},+\infty\left[\right.\right.$ such that $m_{k-1}<t<l$, we have $((\bar{n}, t),(\bar{m}, l)) \in$ $I_{k}\left(\mathbb{M}_{2}\right)$, and

$$
|f(\bar{n}, t)-f(\bar{m}, l)|_{J_{p}^{* *}}^{p} \leq 2^{p} \operatorname{Lip}(f)^{p}+\left(2 \operatorname{Lip}(f)(k-1)^{\frac{1}{p}}+2 \eta\right)^{p}+\eta .
$$

So :

$$
|f(\bar{n}, t)-f(\bar{m}, l)|_{J_{p}^{* *}}^{p} \leq 2^{p} \operatorname{Lip}(f)^{p} k+\varphi(\eta), \text { with } \varphi(\eta) \underset{\eta \rightarrow 0}{\rightarrow} 0 .
$$

Thus, if $\eta$ was chosen small enough :

$$
|f(\bar{n}, t)-f(\bar{m}, l)|_{J_{p}^{* *}} \leq 2 \operatorname{Lip}(f) k^{1 / p}+\varepsilon .
$$

This finishes our inductive proof.

Corollary 3.6. Let $1<q<p<\infty$.

Then $J_{q}$ does not coarse Lipschitz embed into $J_{p}$.

Proof. Suppose that $g: J_{q} \rightarrow J_{p}$ is a map such that there exist $\theta, A$ and $B$ real positive numbers such that :

$$
\forall x, y \in J_{q} \quad\|x-y\|\left\|_{J_{q}} \geq \theta \Rightarrow A\right\| x-y\left\|_{J_{q}} \leq|g(x)-g(y)|_{J_{p}} \leq B\right\| x-y\|\|_{J_{q}} .
$$

Let us rescale by defining $f(v)=A \theta^{-1} g(\theta v)$, for $v \in J_{q}$. We have that there exists $C \geq 1$ such that

$$
\forall x, y \in J_{q},\|x-y\|\left\|_{J_{q}} \geq 1 \Rightarrow\right\| x-y\left\|_{J_{q}} \leq|f(x)-f(y)|_{J_{p}} \leq C\right\| x-y \|_{J_{q}} .
$$

We still denote $\left(e_{n}\right)_{n=1}^{\infty}$ the canonical basis of $J_{q}$.

Consider the map $\varphi: G_{k}(\mathbb{N}) \rightarrow\left(J_{q},\|\|_{J_{q}}\right)$ defined by $\varphi(\bar{n})=e_{n_{1}}+\ldots+e_{n_{k}}$.

Note that $\operatorname{Lip}(\varphi) \leq 4$. Since $\|\varphi(\bar{n})-\varphi(\bar{m})\|_{J_{q}} \geq 1$ whenever $\bar{n} \neq \bar{m}$, we have that $\operatorname{Lip}(f \circ \varphi) \leq 4 C$, where $f \circ \varphi$ is considered as a map from $G_{k}(\mathbb{N})$ to $\left(J_{p},|| J_{p}\right)$. It then follows from Proposition 3.5 that there exist $(\bar{n}, \bar{m}) \in I_{k}(\mathbb{N})$ such that:

$$
|(f \circ \varphi)(\bar{n})-(f \circ \varphi)(\bar{m})|_{J_{p}} \leq 9 C k^{\frac{1}{p}}
$$

On the other hand, since $(\bar{n}, \bar{m}) \in I_{k}(\mathbb{N})$, we have that

$$
\|\varphi(\bar{n})-\varphi(\bar{m})\|_{J_{q}} \geq 2(2 k-1)^{\frac{1}{q}} \geq 2 k^{\frac{1}{q}} .
$$


This is in contradiction with (3.4), for $k$ large enough.

Therefore, there is no coarse Lipschitz embedding from $J_{q}$ into $J_{p}$.

Corollary 3.7. Let $1<p<q<\infty$, and $r>1$ such that $r \notin\{p, q\}$.

Then $J_{r}$ does not coarse Lipschitz embed into $J_{p} \oplus J_{q}$.

Proof. When $r>q$, the argument is based on a midpoint technique like in the proof of Corollary 3.3 ,

If $r<p$, we mimic the proof of Corollary 3.6.

So we assume, as we may, that $1<p<r<q<\infty$ and $f: J_{r} \rightarrow J_{p} \oplus_{\infty} J_{q}$ is a map such that there exists $C \geq 1$ such that

$$
\forall x, y \in J_{r} \quad|x-y|_{J_{r}} \geq 1 \Rightarrow|x-y|_{J_{r}} \leq|| f(x)-f(y) \| \leq C|x-y|_{J_{r}} .
$$

We follow the proof in 8 and write $f=(g, h)$. We still denote $\left(e_{n}\right)_{n=1}^{\infty}$ the canonical basis of $J_{r}$. We fix $k \in \mathbb{N}$ and $\varepsilon>0$. We recall that

$$
\exists \gamma>0, \forall x \in J_{r}, \gamma\|x\|_{J_{r}} \leq|x|_{J_{r}} \leq\|x\|_{J_{r}} .
$$

We start by applying the midpoint technique to the coarse Lipschitz map $g$ and deduce from Proposition 3.2 that there exist $\theta>\gamma^{-1}(2 k)^{1 / r}, u \in J_{r}, N \in \mathbb{N}$ and $K$ a compact subset of $J_{p}$ such that :

$$
g\left(u+\theta B_{\left(E_{N},|\cdot| J_{r}\right)}\right) \subset K+\varepsilon \theta B_{\left(J_{p},|| \cdot \|_{J_{p}}\right)} .
$$

Let $\mathbb{M}=\{n \in \mathbb{N}, n>N\}$ and $\varphi: G_{k}(\mathbb{M}) \mapsto J_{r}$ be defined as follows

$$
\forall \bar{n}=\left(n_{1}, \ldots, n_{k}\right) \in G_{k}(\mathbb{M}), \varphi(\bar{n})=u+\theta(2 k)^{-\frac{1}{r}}\left(e_{n_{1}}+\ldots+e_{n_{k}}\right) .
$$

Then $\varphi(\bar{n}) \in u+\theta B_{\left(E_{N},|\cdot|_{J_{r}}\right)}$ for all $\bar{n} \in G_{k}(\mathbb{M})$.

And, from (3.6) we deduce that $(g \circ \varphi)\left(G_{k}(\mathbb{M})\right) \subset K+\varepsilon \theta B_{\left(J_{p},\|.\| \|_{J_{p}}\right)}$. Thus, by Ramsey's theorem, there is an infinite subset $\mathbb{M}^{\prime}$ of $\mathbb{M}$ such that

$$
\operatorname{diam}_{\|\|_{J_{p}}}(g \circ \varphi)\left(G_{k}\left(\mathbb{M}^{\prime}\right)\right) \leq 3 \varepsilon \theta .
$$

Since for $\bar{n} \neq \bar{m}$, we have

$$
|\varphi(\bar{n})-\varphi(\bar{m})|_{J_{r}} \geq \gamma|| \varphi(\bar{n})-\left.\varphi(\bar{m})\right|_{J_{r}} \geq \gamma \theta(2 k)^{-\frac{1}{r}}>1,
$$

it follows from (3.5) that

$\forall \bar{n}, \bar{m} \in G_{k}(\mathbb{M}) \quad\|h \circ \varphi(\bar{n})-h \circ \varphi(\bar{m})\|_{J_{q}} \leq C|\varphi(\bar{n})-\varphi(\bar{m})|_{J_{r}} \leq C\|\varphi(\bar{n})-\varphi(\bar{m})\|_{J_{r}}$.

Note now that for all $\bar{n}, \bar{m} \in G_{k}(\mathbb{N})$,

$$
\left\|\left(e_{n_{1}}+\ldots+e_{n_{k}}\right)-\left(e_{m_{1}}+\ldots+e_{m_{k}}\right)\right\|_{J_{q}} \leq \sum_{n_{i} \neq m_{i}}\left\|e_{n_{i}}-e_{m_{i}}\right\|_{J_{q}} \leq 2 d(\bar{n}, \bar{m}) .
$$

Since moreover ||$_{J_{q}} \leq\|\|_{J_{q}}, \operatorname{Lip}(h \circ \varphi) \leq 2 C \theta(2 k)^{-\frac{1}{r}}$, when $h \circ \varphi$ is considered as a map from $G_{k}\left(\mathbb{M}^{\prime}\right)$ to $\left(J_{q},||_{J_{q}}\right)$. Thus, we can apply Proposition 3.5 to obtain:

$$
\exists(\bar{n}, \bar{m}) \in I_{k}\left(\mathbb{M}^{\prime}\right),|h \circ \varphi(\bar{n})-h \circ \varphi(\bar{m})|_{J_{q}} \leq 5 C \theta(2 k)^{-\frac{1}{r}} k^{\frac{1}{q}} .
$$


Then, if $k$ was chosen large enough, we have:

$$
\exists(\bar{n}, \bar{m}) \in I_{k}\left(\mathbb{M}^{\prime}\right),|h \circ \varphi(\bar{n})-h \circ \varphi(\bar{m})|_{J_{q}} \leq \varepsilon \theta .
$$

This, combined with (3.7) implies that

$$
\exists(\bar{n}, \bar{m}) \in I_{k}\left(\mathbb{M}^{\prime}\right) \quad\|f \circ \varphi(\bar{n})-f \circ \varphi(\bar{m})\| \leq 3 \varepsilon \theta .
$$

But,

$$
\forall(\bar{n}, \bar{m}) \in I_{k}\left(\mathbb{M}^{\prime}\right) \quad|\varphi(\bar{n})-\varphi(\bar{m})|_{J_{r}} \geq \gamma\|\varphi(\bar{n})-\varphi(\bar{m})\|_{J_{r}} \geq \gamma \theta .
$$

If $\varepsilon$ was initially chosen such that $\varepsilon<\frac{\gamma}{3}$, this yields a contradiction with (3.5), which concludes our proof.

Remark. This result can be easily extended as follows. Assume $r \in(1, \infty) \backslash$ $\left\{p_{1}, \ldots, p_{n}\right\}$ where $1<p_{1}<p_{2}<\ldots<p_{n}<\infty$, then $J_{r}$ does not coarse Lipschitz embed into $J_{p_{1}} \oplus \ldots \oplus J_{p_{n}}$.

\section{AKnowledgment}

The author is grateful to Gilles Lancien for sharing his knowledge and experience, and for helpful discussions that contributed to shape this paper.

\section{REFERENCES}

[1] Y. Benyamini and J. Lindenstrauss, Geometric nonlinear functionan analysis, A.M.S. Colloquium publications, vol 48, American Mathematical Society, Providence, RI, (2000).

[2] J. Bourgain, Remarks on the extension of Lipschitz maps defined on discrete sets and uniform homeomorphisms, Geometrical aspects of functional analysis (1985/86), Lecture Notes in Math., 1267, Springer Berlin, (1987), 157-167.

[3] E. Gorelik, The uniform nonequivalence of $L_{p}$ and $l_{p}$, Israel J. Math, 87, (1994), 1-8.

[4] W.T. Gowers, Ramsey methods in Banach spaces, Handbook of the geometry of Banach spaces, vol 2, North-Holland, Amsterdam, (2003), 10711097.

[5] R.C. James, A somewhat relexive Banach space with non separable dual, Bull. A.M.S. 80, (1974), 738-743.

[6] W.B. Johnson, J. Lindenstrauss and G. Schechtman, Banach spaces determined by their uniform structures, Geom. Funct. Anal., 6, (1996), 430-470.

[7] N.J. Kalton, Coarse and uniform embeddings into reflexive spaces, Quart. J. Math. (Oxford), 58(1), (2007), 393-414.

[8] N.J. Kalton, N.L. Randrianarivony, The coarse Lipschitz structure of $\ell_{p} \oplus \ell_{q}$, Math. Ann., 341, (2008), 223-237.

[9] G. Lancien, A short course on non linear geometry of Banach spaces. Topics in Functional and Harmonic Analysis, Theta Series in Advanced Mathematics, (2012), 77-102.

[10] G. Lancien, Réflexivité et normes duales possédant la propriété uniforme de Kadec-Klee, Publ. Math. de la Fac. des sciences de Besançon, Fascicule 14, (1993-94), 69-74.

[11] G. Pisier, The dual $J^{*}$ of the James Space has cotype 2 and the Gordon-Lewis property, Proc. Cambridge Phil., 103, (1988), 323-331.

Université de Franche-Comté, Laboratoire de Mathématiques UMR 6623, 16 route de Gray, 25030 Besançon Cedex, FRAnCE.

E-mail address: francois.netillard@univ-fcomte.fr 